\title{
Trends and Growth Performance in Area, Production and Productivity of Four Common Major Crops of Pakistan and India
}

\author{
Dr. Mohammad Pervez Wasim
}

\begin{abstract}
The study analyzed and compared the growth rates of area, production and productivity of common major crops of Pakistan and India from 198485 to 2008 09. It reveals that in Pakistan the increase in the production growth rate of rice, wheat and sugarcane were due to increase both in its area and productivity, but productivity growth rate contributed more as compared to increase in its area growth, while for cottonseed area and productivity growth contributed equally. The study also confirms that in India the increase in the production growth of rice and wheat were due to increase both in its area and productivity growth but productivity contributed more than area. The study also confirms that area growth rate of rice and cottonseed for Pakistan increased by more percentage per annum as compared to India. The study also concludes that the area growth rate of wheat and sugarcane increased by more percentage for India as compared to Pakistan. The major finding of this study is that as far as production and productivity growth rates are concerned, it increased by more percentage for all the crops of Pakistan as compared to Indian crops.
\end{abstract}

Keywords: Growth rates, technology-based growth in productivity, research and extension services, favorable price incentives, HYVs, negative area positive productivity.

\section{Introduction}

It is needless to mention the role played by agriculture in developing countries like Pakistan and India. Majority of the population in both the countries are rural based and relies on employment and income, which depends in one way or another on agriculture. Therefore, agricultural growth is a catalyst for economic growth and development of the country. Its contribution towards capital formation etc., are well known. Its linkages with industries in supplying wage goods, providing raw materials and creating demand for the industrial products are well documented in literature.

Therefore a stable growth in crops area, production and productivity for Pakistan and India are highly essential.

Some of the studies (Narain (1977), Ray (1983), Parthasarathy (1984), Rao and Deshpande (1986), Mahendradev (1987), Thakur, Singh and Roy (1988), Pal and Sirohi (1988), Mitra (1990) and Swant and Achuthan (1995) related to this topic are available for India. In Pakistan, analysis of growth in crop production has been limited to only few studies (Khan and Siddiqui (1982), Khan and Mohsin (1995), Ghaffar, Mustafa and

Dr. Mohammad Pervez Wasim is Research Economist at Applied Economics Research Centre, (AERC), University of Karachi.

Journal of Independent Studies and Research - MSSE

Volume $9 \quad$ Number 1

January $2011 \mid 125$ 
Qasim (1996) and Wasim (1999). However, no attempt has been made to estimate the growth differentials in area, production and productivity of rice, wheat, cotton seed and sugarcane crops for Pakistan and India.

Agriculture still generated 45 and 52 per cent of Pakistan's and India's employment respectively in 200708 . It contributed about 21.8 and 16.6 per cent respectively of the Gross Domestic Product (GDP) in 200809.

In most of the crops there are per acre productivity differentials between both the countries. Better management of input supplies, lively policy and adoption of scientific agronomic and other cultural practices, sowing crops well in time, appropriate doses of fertilizer and water, better weed management practices, developments of rural roads, electricity and tubewells, tractorization, well-knit agricultural education and extension system played a vital role in productivity differential.

The magnitude of growth in area, production and productivity has serious implication for food security in Pakistan and India. Information about productivity growth differentials in major crops would help the policy makers of Pakistan and India to implement policies to increase productivity per hectare and export-import policy for different agricultural crops.

Examination of the issues stated above is expected to throw light on the nature of differential in crop productivity.

The present study is undertaken with a view to analyze the trends and growth situation in four major crops area, production and productivity of Pakistan and India. The specific objectives of the study are as follows:

1. to discuss about trends in area, production and productivity per hectare of rice, wheat, cotton seed and sugarcane in Pakistan and India.

2. to discuss about crops production share of Pakistan and India in their respective world crop production from 1995-96 to 2008-09.

3. to make a comparison of Pakistani and Indian crops productivity per hectare with respect to world productivity per hectare, $1995-96$ to 2008-09.

4. to quantify the rate of growth in area, production and productivity of crops separately for Pakistan and India and within Pakistan and India.

5. to discuss the policy implications of the observed growth scenario in major crops production and productivity.

\section{Data Sources}

The analysis is based on secondary data for the last 25 years, i.e. from 198485 to 200809 . The data related to area, production and productivity of rice, wheat, cottonseed and sugarcane crops of Pakistan and India were obtained from the Agricultural Statistics of Pakistan, Ministry of Food, Agriculture and Livestock, Government of Pakistan and 


\section{Economic Survey, Finance Division, Government of Pakistan.}

\section{Research Methodology}

The annual compound growth rates are estimated by using loglinear functions on the time series data on area, production and productivity. The equation fitted to analyze the trend growth rate is semilog exponential form.

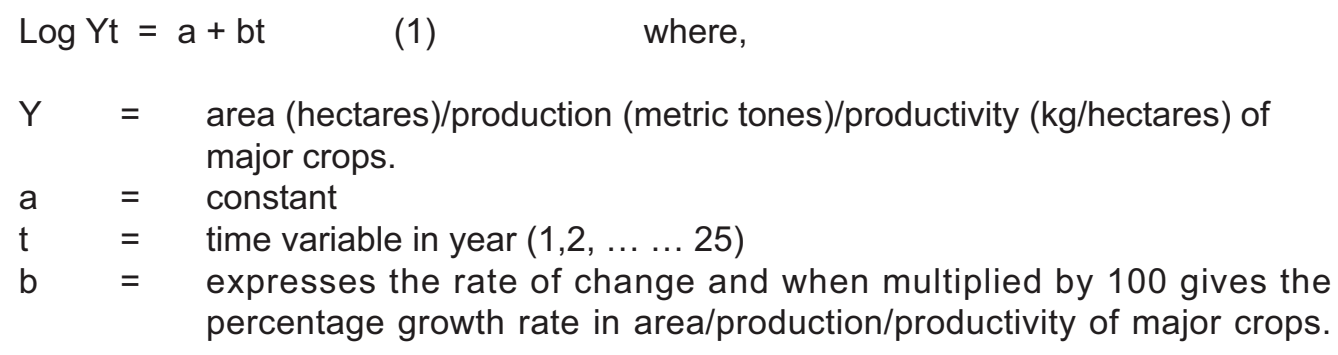

where,

$\mathrm{Y}=$ area (hectares)/production (metric tones)/productivity $(\mathrm{kg} /$ hectares) of major crops.

$\mathrm{a}=$ constant

$\mathrm{t}=$ time variable in year $(1,2, \ldots \ldots 25)$

$\mathrm{b}=$ expresses the rate of change and when multiplied by 100 gives the percentage growth rate in area/production/productivity of major crops.

\section{Results and Discussion}

Trends in Area, Production and Productivity of Crops

\section{Pakistan}

Trends in area, production and productivity are presented in Table 1 and Graph 1.

\section{Rice}

The area under rice showed an increasing trend, being 1998 thousand hectares at the starting of the period and ended to 2963 thousand hectares at the end of the study period (2008 09). The maximum area of 2963 thousand hectares was recorded in 2008 09. Production and productivity of the crop showed more or less an increasing trend. The production of the crop showed three times increase at the end of the study period. The productivity of the crop recorded its highest (3520 Kg./hectares) in 2008 09. Per hectare increase in productivity was mainly due to favorable climatic conditions and adoption of new farm technologies. 
Table - 1 Trends in Area, Production and Productivity of Major Crops in Pakistan

\begin{tabular}{|c|c|c|c|c|c|c|c|c|c|c|c|c|}
\hline \multirow[b]{2}{*}{ Ye ars } & \multicolumn{2}{|r|}{ Rice } & \multicolumn{4}{|c|}{ Wheat } & \multicolumn{3}{|c|}{ Cotton Seed } & \multicolumn{3}{|c|}{ Sugarcane } \\
\hline & $\begin{array}{c}\text { Area } \\
\text { ('000 } \\
\text { hectar } \\
\text { es) }\end{array}$ & $\begin{array}{l}\text { Prodn. } \\
\text { ('000 } \\
\text { m.tonn } \\
\text { es) }\end{array}$ & $\begin{array}{l}\text { Prody. } \\
\text { (Kg/ } \\
\text { hectar } \\
\text { es) }\end{array}$ & $\begin{array}{c}\text { Area } \\
\text { ('000 } \\
\text { hectar } \\
\text { es) }\end{array}$ & $\begin{array}{c}\text { Prodn. } \\
\text { ('000 } \\
\text { m.tonn } \\
\text { es) }\end{array}$ & $\begin{array}{l}\text { Prody. } \\
\text { (Kg/ } \\
\text { hectar } \\
\text { es) }\end{array}$ & $\begin{array}{c}\text { Area } \\
\text { ('000 } \\
\text { hectar } \\
\text { es) }\end{array}$ & $\begin{array}{l}\text { Prodn. } \\
\text { ('000 } \\
\text { m.tonn } \\
\text { es) }\end{array}$ & $\begin{array}{l}\text { Prody. } \\
\text { (Kg/ } \\
\text { hectar } \\
\text { es) }\end{array}$ & $\begin{array}{c}\text { Area } \\
\text { ('000 } \\
\text { hectar } \\
\text { es ) }\end{array}$ & $\begin{array}{l}\text { Prodn. } \\
\text { ('000 } \\
\text { m.tonn } \\
\text { es) }\end{array}$ & $\begin{array}{l}\text { Prody. } \\
\text { (Kg/ } \\
\text { hectar } \\
\text { es) }\end{array}$ \\
\hline $1984-85$ & 1998 & 3340 & 1672 & 7343 & 10882 & 1481 & 2242 & 3026 & 1350 & 896 & 34287 & 38267 \\
\hline $1985-86$ & 1998 & 3315 & 1659 & 7259 & 11703 & 1613 & 2364 & 3651 & 1545 & 904 & 32140 & 35553 \\
\hline $1986-87$ & 1863 & 2919 & 1567 & 7403 & 13923 & 1881 & 2505 & 3959 & 1580 & 780 & 27856 & 35713 \\
\hline $1987-88$ & 2066 & 3486 & 1687 & 7706 & 12016 & 1559 & 2568 & 4405 & 1716 & 762 & 29926 & 39273 \\
\hline $1988-89$ & 1963 & 3241 & 1651 & 7308 & 12675 & 1734 & 2619 & 4278 & 1632 & 842 & 33029 & 39227 \\
\hline $1989-90$ & 2042 & 3200 & 1567 & 7730 & 14419 & 1865 & 2599 & 4367 & 1680 & 877 & 36976 & 42162 \\
\hline $1990-91$ & 2107 & 3220 & 1528 & 7845 & 14316 & 1825 & 2662 & 4913 & 1845 & 854 & 35494 & 41562 \\
\hline 1991-92 & 2113 & 3261 & 1543 & 7911 & 14565 & 1841 & 2836 & 6543 & 2307 & 884 & 35989 & 40711 \\
\hline $1992-93$ & 2097 & 3243 & 1546 & 7878 & 15684 & 1991 & 2836 & 4620 & 1629 & 896 & 38865 & 43376 \\
\hline $1993-94$ & 1973 & 3116 & 1579 & 8300 & 16157 & 1946 & 2805 & 4103 & 1464 & 885 & 38059 & 43004 \\
\hline 1994-95 & 2187 & 3995 & 1827 & 8034 & 15213 & 1893 & 2653 & 4438 & 1671 & 963 & 44427 & 46134 \\
\hline $1995-96$ & 2125 & 3447 & 1622 & 8170 & 17002 & 2081 & 2997 & 4783 & 1596 & 1009 & 47168 & 46747 \\
\hline $1996-97$ & 2162 & 3967 & 1835 & 8377 & 16907 & 2018 & 3149 & 4686 & 1584 & 963 & 45230 & 46968 \\
\hline 1997-98 & 2251 & 4305 & 1912 & 8109 & 16651 & 2053 & 2960 & 4686 & 1584 & 965 & 41998 & 43521 \\
\hline $1998-99$ & 2317 & 4333 & 1870 & 8395 & 18694 & 2238 & 2923 & 4485 & 1536 & 1050 & 53104 & 50575 \\
\hline $\begin{array}{c}1999- \\
2000\end{array}$ & 2424 & 4674 & 1928 & 8230 & 17856 & 2170 & 2960 & 5735 & 1962 & 1155 & 55191 & 47780 \\
\hline $2000-01$ & 2515 & 5156 & 2050 & 8463 & 21079 & 2491 & 2927 & 5476 & 1871 & 1010 & 46333 & 45883 \\
\hline 2001-02 & 2376 & 4803 & 2021 & 8125 & 19024 & 2341 & 2794 & 5476 & 1960 & 961 & 43606 & 45385 \\
\hline $2002-03$ & 2225 & 6718 & 3019 & 8058 & 18227 & 2262 & 2989 & 5187 & 1735 & 1000 & 48042 & 48056 \\
\hline 2003-04 & 2461 & 7272 & 2955 & 8034 & 19183 & 2388 & 3100 & 5197 & 1676 & 1100 & 52056 & 47323 \\
\hline 2004-05 & 2520 & 7538 & 2991 & 8358 & 21612 & 2586 & 2989 & 5127 & 1715 & 1075 & 53419 & 49692 \\
\hline 2005-06 & 2621 & 8321 & 3174 & 8448 & 21277 & 2519 & 3193 & 7279 & 2280 & 966 & 47244 & 48907 \\
\hline $2006-07$ & 2572 & 8137 & 3164 & 8494 & 23520 & 2769 & 3075 & 6252 & 2033 & 907 & 44666 & 49229 \\
\hline 2007-08 & 2600 & 8300 & 3192 & 8578 & 23295 & 2716 & 3054 & 5677 & 1859 & 1029 & 54752 & 53209 \\
\hline 2008-09 & 2963 & 10428 & 3520 & 8550 & 20959 & 2451 & 2820 & 5770 & 2046 & 1241 & 63920 & 51494 \\
\hline
\end{tabular}

Source: Agricultural Statistics of Pakistan (various issues). 

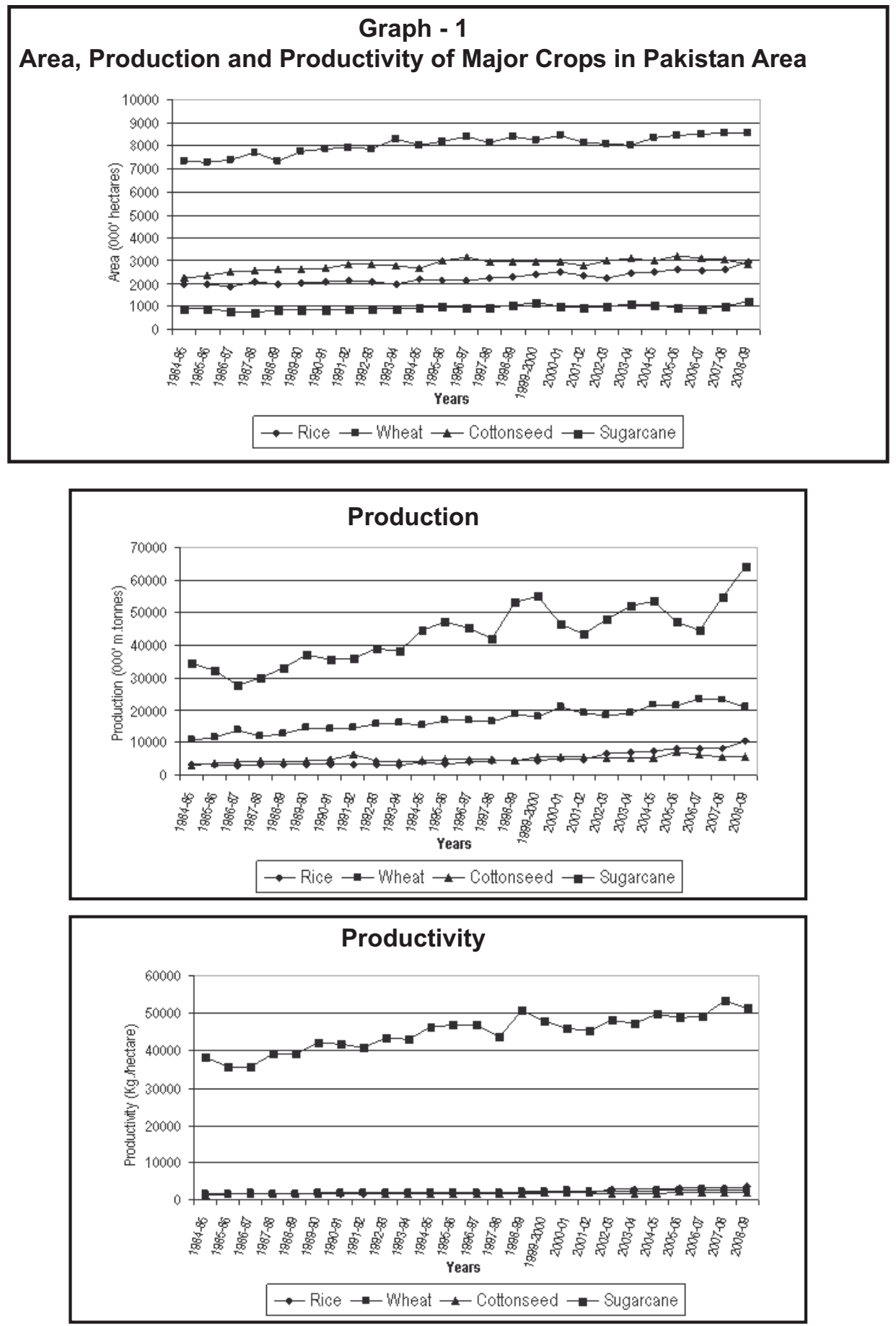


\section{Wheat}

Area, production and productivity of the crop recorded fluctuating but increasing trend. Production and productivity just doubled at the end of the study period as compared to area. It clearly indicates the utilization of new farm technologies.

\section{Cotton Seed}

The area, production and productivity of the crop showed a combination of fluctuating and increasing trend. Production and productivity of the crop increased by more percentage as compared to area increase. The highest area of 3193 thousand hectares was recorded in 2005 06, highest production of 7279 thousand tonnes was recorded in 2005-06 and highest yield of $2307 \mathrm{~kg}$ per hectare was in 1991-92.

\section{Sugarcane}

Though the crop area, production and productivity increased at the end of the study period but with a fluctuating trend. Production and productivity increased by more percentage as compared to area. The highest production was recorded in 200809.

India

Trends in area, production and productivity are presented in Table 2 and Graph 2. 
Table - 2 Trends in Area, Production and Productivity of Major Crops in India

\begin{tabular}{|c|c|c|c|c|c|c|c|c|c|c|c|c|}
\hline \multirow[b]{2}{*}{ Years } & \multicolumn{2}{|c|}{ Rice } & \multicolumn{4}{|c|}{ Wheat } & \multicolumn{3}{|c|}{ Cotton Seed } & \multicolumn{3}{|c|}{ Sugarcane } \\
\hline & $\begin{array}{c}\text { Area } \\
\text { ('000 } \\
\text { hectar } \\
\text { es) }\end{array}$ & $\begin{array}{c}\text { Prodn. } \\
\text { ('000 } \\
\text { m.tonn } \\
\text { es) }\end{array}$ & $\begin{array}{c}\text { Prody. } \\
\text { (Kg/ } \\
\text { hectar } \\
\text { es) }\end{array}$ & $\begin{array}{c}\text { Area } \\
\text { ('000 } \\
\text { hectar } \\
\text { es) }\end{array}$ & $\begin{array}{l}\text { Prodn. } \\
\text { ('000 } \\
\text { m.tonn } \\
\text { es) }\end{array}$ & $\begin{array}{c}\text { Prody. } \\
\text { (Kg/ } \\
\text { hectar } \\
\text { es) }\end{array}$ & $\begin{array}{c}\text { Area } \\
\text { ('000 } \\
\text { hectar } \\
\text { es) }\end{array}$ & $\begin{array}{l}\text { Prodn. } \\
\text { ('000 } \\
\text { m.tonn } \\
\text { es) }\end{array}$ & $\begin{array}{c}\text { Prody. } \\
\text { (Kg/ } \\
\text { hectar } \\
\text { es) }\end{array}$ & $\begin{array}{c}\text { Area } \\
\text { ('000 } \\
\text { hectar } \\
\text { es) }\end{array}$ & \begin{tabular}{|c|} 
Prodn. \\
('000 \\
m.tonn \\
es)
\end{tabular} & $\begin{array}{l}\text { Prody. } \\
\text { (Kg/ } \\
\text { hectar } \\
\text { es) }\end{array}$ \\
\hline 1984-85 & 41200 & 60100 & 1459 & 24700 & 45500 & 1842 & 7721 & 6386 & 827 & 3110 & 174000 & 55948 \\
\hline $1985-86$ & 41200 & 58300 & 1415 & 23600 & 44100 & 1869 & 7382 & 8507 & 1152 & 2953 & 170000 & 57568 \\
\hline $1986-87$ & 40900 & 64200 & 1570 & 23100 & 46900 & 2030 & 7581 & 8612 & 1136 & 2862 & 172000 & 60098 \\
\hline $1987-88$ & 38319 & 60600 & 1581 & 23300 & 44300 & 1901 & 7031 & 6905 & 982 & 3202 & 186000 & 58089 \\
\hline $1988-89$ & 41736 & 56900 & 1363 & 23500 & 46200 & 1966 & 7273 & 6382 & 877 & 3475 & 197000 & 56690 \\
\hline $1989-90$ & 42177 & 70500 & 1671 & 23900 & 54100 & 2263 & 7690 & 5864 & 762 & 3489 & 203000 & 58183 \\
\hline $1990-91$ & 41800 & 73600 & 1761 & 23500 & 49800 & 2119 & 7695 & 5029 & 653 & 3411 & 226000 & 66256 \\
\hline $1991-92$ & 41927 & 74300 & 1772 & 24200 & 55100 & 2277 & 7440 & 4981 & 665 & 3682 & 241000 & 65453 \\
\hline $1992-93$ & 41637 & 74700 & 1794 & 23300 & 55700 & 2390 & 7661 & 5937 & 775 & 4105 & 249000 & 64776 \\
\hline 1993-94 & 42034 & 72600 & 1727 & 24600 & 57200 & 2325 & 7543 & 6274 & 832 & 4112 & 228000 & 63018 \\
\hline 1994-95 & 41457 & 72600 & 1751 & 24900 & 59100 & 2373 & 7321 & 7039 & 961 & 3442 & 227000 & 63443 \\
\hline $1995-96$ & 42500 & 72400 & 1703 & 25400 & 63000 & 2480 & 7871 & 7141 & 907 & 3867 & 259000 & 69067 \\
\hline $1996-97$ & 43280 & 77000 & 1779 & 25000 & 62100 & 2484 & 9035 & 7276 & 805 & 4147 & 282900 & 68218 \\
\hline $1997-98$ & 43080 & 81700 & 1896 & 25900 & 69400 & 2679 & 9121 & 7913 & 867 & 4174 & 277250 & 66423 \\
\hline 1998-99 & 42300 & 82500 & 1950 & 26700 & 66300 & 2483 & 8868 & 7720 & 871 & 3930 & 289000 & 73537 \\
\hline $\begin{array}{l}1999- \\
2000\end{array}$ & 44800 & 86100 & 1922 & 27500 & 71300 & 2593 & 8759 & 5910 & 675 & 4055 & 289000 & 71270 \\
\hline 2000-01 & 45000 & 89500 & 1989 & 27400 & 75600 & 2759 & 8409 & 4788 & 890 & 4225 & 299000 & 70769 \\
\hline 2001-02 & 44500 & 92300 & 2074 & 25000 & 68500 & 2740 & 8800 & 5250 & 596 & 4050 & 286000 & 70617 \\
\hline $2002-03$ & 40410 & 108900 & 2695 & 26340 & 72760 & 2762 & 7700 & 4450 & 578 & 4412 & 297208 & 67370 \\
\hline 2003-04 & 44000 & 130400 & 2964 & 24886 & 65120 & 2617 & 7800 & 7040 & 903 & 4608 & 281600 & 61116 \\
\hline 2004-05 & 42500 & 124400 & 2927 & 27300 & 72060 & 2640 & 8700 & 7200 & 828 & 4100 & 244800 & 59707 \\
\hline $2005-06$ & 43400 & 130513 & 3007 & 26500 & 72000 & 2717 & 8826 & 7500 & 850 & 3750 & 232320 & 61952 \\
\hline 2006-07 & 43617 & 139137 & 3190 & 26484 & 69355 & 2619 & 9142 & 11568 & 1265 & 4201 & 281172 & 66928 \\
\hline 2007-08 & 44000 & 141134 & 3208 & 28035 & 74890 & 2671 & 9300 & 9480 & 1019 & 4900 & 355200 & 72555 \\
\hline 2008-09 & 44000 & 148260 & 3370 & 28039 & 78570 & 2802 & 9373 & 11305 & 1206 & 5055 & 348188 & 68877 \\
\hline
\end{tabular}

Source: Agricultural Statistics of Pakistan (various issues). 

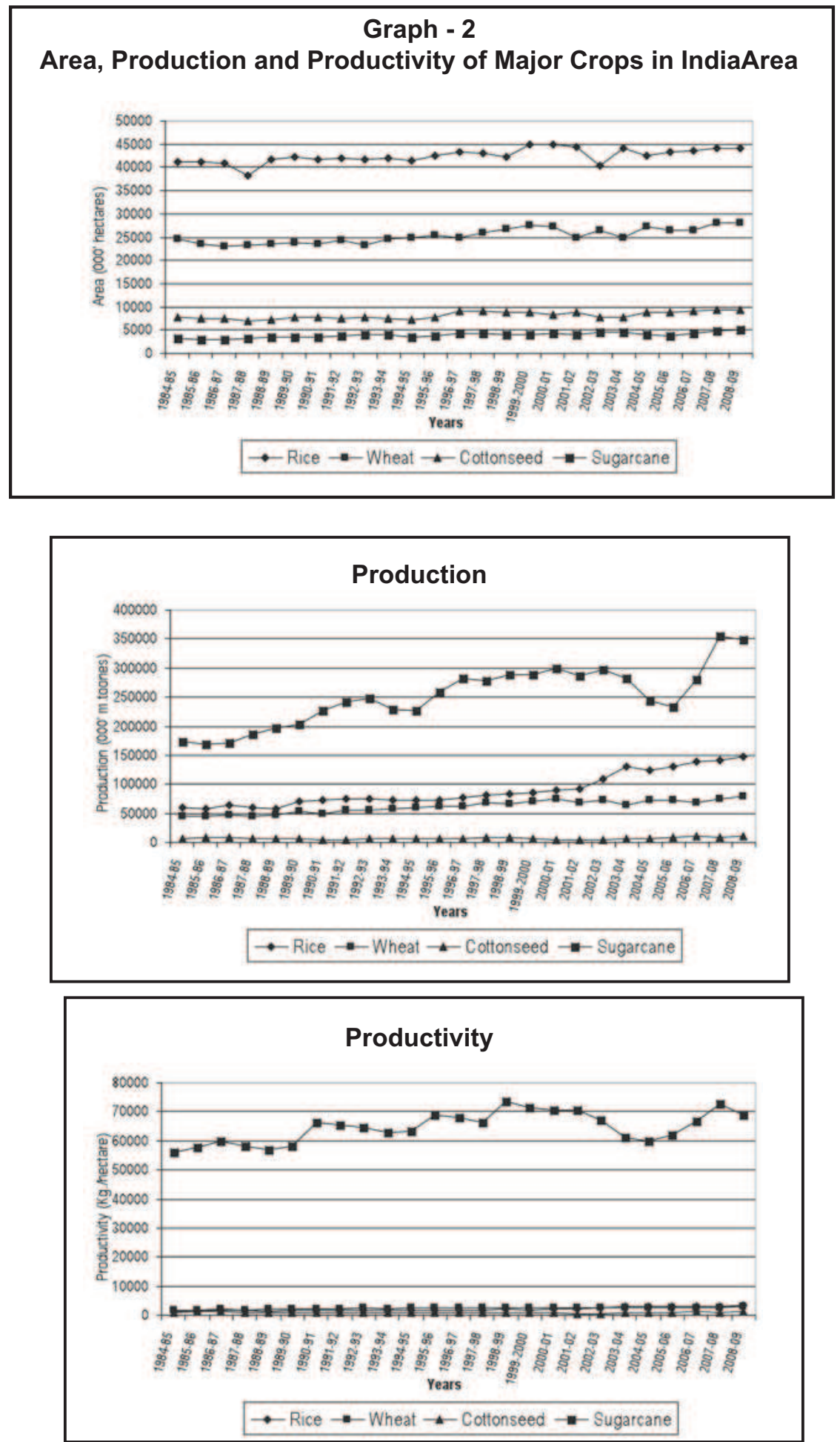


\section{Rice}

Madhya Pradesh. Production and productivity of the crop increased more as compared to increase in its area. The maximum production and productivity was recorded at the end of the study period (2008 09), while area recorded highest hectarage in 2000-01.

\section{Wheat}

Uttar Pradesh, Punjab, Haryana and Madhya Pradesh are the major growing states for wheat cultivation. Production and productivity of the crop increased more as compared to increase in its area. The highest production and producivity was recorded in 200809.

\section{Cotton Seed}

Mostly grown in the state of Punjab, Andhra Pradesh and Maharashtra. The production and productivity of the crop increased from 6386 thousand tonnes and $827 \mathrm{~kg}$ per hectare respectively in 1984-85 to 11305 thousand tonnes and $1206 \mathrm{~kg}$ per hectare respectively in 2008-09. The area of the crop increased slightly. The highest productivity of $1265 \mathrm{~kg}$ per hectares was recorded in the year 2006-07.

\section{Sugarcane}

The area, production and productivity of the crop increased at the end of the study period, though this increase showed a fluctuating trend. Uttar Pradesh is the major state for sugercane cultivation. The highest increase in production and productivity was recorded in 200708.

\section{Common Major Crops Production Share Of Pakistan And India In Their Respective World Crop Production, 1995-96 To 2008-09}

We have also calculated Pakistan's and India's common major crops production share in their respective crops world production. India has the large percentage of world production share in all the crops production as compared to Pakistan mainly because India has large each crop acreage as compared to Pakistan. India had the maximum world production share (23.84 per cent) in sugarcane, followed by rice (22.24 per cent), cotton seed (17.13 percent) and wheat (12.95 per cent). From 1995-96 to 2008-09 shares are shown in Table 3 and Bar-Diagram 3. As far as Pakistan is concerned it has highest percentage world share (10.85 per cent) in cottonseed, followed by sugarcane (4.36 per cent), wheat (3.88 per cent) and rice ( 1.52 per cent). If we compare the two countries' crops production share, then it can be seen that since India has more cultivated area of each crop as compared to Pakistan, its world crop production share is also high for all common crops. Pakistan has the highest world crop production share in cottonseed while India has in sugarcane. Pakistan and India has minimum world share in rice and wheat respectively. 


\section{Comparison Of Productivity Per Hectare Of Crops Of Pakistan And India With Respective To Crops World Productivity, 199596 To 2008 --09}

Productivity per hectares means that how many of kilograms of any crop you are getting from one hectare of land. Higher productivity of any crop means that the factors like favorable price incentives, expanded irrigation system, good monsoons rain (in case of India), HYVs, rising level of chemical fertilizer applications, timely sowing, better use

\section{Productivity}

Table - 3 Common Major Crops Production Share of Pakistan and India in Their Respective World Crop Production, 1995-96 to 2008-09

\begin{tabular}{|c|c|c|c|c|c|c|c|c|c|c|c|c|c|c|c|c|c|c|c|c|}
\hline \multirow[b]{3}{*}{ Years } & \multicolumn{5}{|c|}{ Rice } & \multicolumn{5}{|c|}{ Wheat } & \multicolumn{5}{|c|}{ Cottonseed } & \multicolumn{5}{|c|}{ Sugarcane } \\
\hline & \multirow{2}{*}{\begin{tabular}{|c|} 
World \\
Produc \\
tion \\
$\left(000^{\prime}\right.$ tones $)$ \\
\cline { 2 - 2 }
\end{tabular}} & \multicolumn{2}{|c|}{ Pakistan } & \multicolumn{2}{|c|}{ India } & \multirow{2}{*}{\begin{tabular}{|c|} 
World \\
Produc \\
tion \\
$\left(000^{\prime}\right.$ tones $)$
\end{tabular}} & \multicolumn{2}{|c|}{ Pakistan } & \multicolumn{2}{|c|}{ India } & \multirow{2}{*}{\begin{tabular}{|c|} 
World \\
Produc \\
tion \\
$\left(000^{\prime}\right.$ tones $)$
\end{tabular}} & \multicolumn{2}{|c|}{ Pakistan } & \multicolumn{2}{|c|}{ India } & \multirow{2}{*}{\begin{tabular}{|c|} 
World \\
Produc \\
tion \\
$\left(000^{\prime}\right.$ tones $)$
\end{tabular}} & \multicolumn{2}{|c|}{ Pakistan } & \multicolumn{2}{|c|}{ India } \\
\hline & & \begin{tabular}{|c|} 
Produc \\
tion \\
$1000^{\circ}$ tones \\
\end{tabular} & $\begin{array}{l}\text { World } \\
\text { Share } \\
(\%)\end{array}$ & $\begin{array}{c}\text { Produc } \\
\text { tion } \\
\left(1000^{\prime} \text { tones }\right)\end{array}$ & $\begin{array}{l}\text { World } \\
\text { Share } \\
(\%)\end{array}$ & & \begin{tabular}{|c|} 
Produc \\
tion \\
(100' tones \\
\end{tabular} & $\begin{array}{l}\text { World } \\
\text { Share } \\
(\%)\end{array}$ & $\begin{array}{c}\text { Produc } \\
\text { tion } \\
\left(000^{\prime} \text { tones) }\right.\end{array}$ & $\begin{array}{l}\text { World } \\
\text { Share } \\
(\%)\end{array}$ & & \begin{tabular}{|c|} 
Produc \\
tion \\
$1000^{\prime}$ tones
\end{tabular} & $\begin{array}{c}\text { World } \\
\text { Share } \\
(\%)\end{array}$ & $\begin{array}{c}\text { Produc } \\
\text { tion } \\
(\text { too' tones) }\end{array}$ & \begin{tabular}{|c|}
$\begin{array}{c}\text { World } \\
\text { Share } \\
(\%)\end{array}$ \\
\end{tabular} & & \begin{tabular}{|c|} 
Produc \\
tion \\
$\left(000^{\prime}\right.$ tones \\
\end{tabular} & & \begin{tabular}{|c|} 
Produc \\
tion \\
$\left(100^{\circ}\right.$ tones $)$
\end{tabular} & \begin{tabular}{|c} 
World \\
Share \\
$(\%)$
\end{tabular} \\
\hline 1995-96 & 550193 & 3447 & 0.63 & 72400 & 13.16 & 578764 & 17002 & 2.94 & 63000 & 10.88 & 57244 & 4783 & 8.35 & 7141 & 12.47 & 1147992 & 47168 & & 59000 & 22.5 \\
\hline 1996-97 & 9236 & 3967 & 0.70 & 77000 & 13.53 & 582689 & 16907 & 2.90 & 62100 & 10.66 & 54793 & 4686 & 8.55 & 7276 & 13.28 & 1226666 & 45230 & 3.69 & 282900 & 23.06 \\
\hline $1997-98$ & 0202 & 4305 & & 81700 & & 12380 & 16651 & & 69400 & 11.33 & 56842 & 4686 & 8.24 & 7913 & 13.92 & 1241394 & 41998 & 3.38 & 77250 & 22.33 \\
\hline 1998-99 & 3188 & 4333 & 0.77 & 82500 & 14.65 & 88842 & 18694 & 3.17 & 66300 & 11.26 & 51793 & 4485 & 8.65 & 7720 & 14.90 & 1252266 & 53104 & 4.24 & 89000 & 23.08 \\
\hline $\begin{array}{l}1999- \\
2000\end{array}$ & 609902 & 4674 & 0.77 & 86100 & 14.12 & 587029 & 17856 & 3.04 & 71300 & 12.14 & 52844 & 5735 & 10.85 & 5910 & \begin{tabular}{|l|l|} 
& 11.18 \\
\end{tabular} & 1264749 & 55191 & 4.36 & 289000 & 22.85 \\
\hline $2000-01$ & 8243 & 5156 & 0.86 & 89500 & 14.96 & 83601 & 21079 & & 75600 & 12.95 & 52928 & 5476 & 10.3 & 4788 & 9.05 & 1254209 & 46333 & & 299000 & 23.84 \\
\hline 2001-02 & 585148 & 4803 & 0.82 & 92300 & 15.77 & 575842 & 19024 & 3.30 & 68500 & 11.89 & 58378 & 5476 & 9.38 & 5250 & 9.00 & 1246845 & 43606 & 3.50 & 286000 & 22.94 \\
\hline 2002-03 & 1076 & 6718 & 1.18 & 108900 & 19.07 & 573967 & 18227 & 3.1 & 72766 & 12.67 & 52802 & 5187 & 9.82 & 4450 & 8.42 & 1339425 & 48042 & 3.58 & 297208 & 22.18 \\
\hline 2003-04 & 36248 & 7272 & 1.24 & 130400 & 22.24 & 557503 & 19183 & 3.44 & 65120 & 11.68 & 55515 & 5197 & 9.36 & 7040 & 12.68 & 1351902 & 52056 & .85 & 281600 & 20.82 \\
\hline 2004-05 & 08496 & 7538 & 1.23 & 124420 & 20.44 & 324093 & 21612 & 3.46 & 72060 & 11.54 & 67375 & 5127 & 7.60 & 7200 & 10.68 & 1318178 & 53419 & 4.05 & 244800 & 18.57 \\
\hline 2005-06 & 18224 & 8321 & 1.34 & 130513 & 21.11 & 631319 & 21277 & 3.37 & 72000 & 11.40 & 68298 & 7279 & 10.65 & 7500 & 10.98 & 1290556 & 47244 & 3.66 & 232320 & 18.00 \\
\hline 2006-07 & 1080 & 8137 & 1.26 & 139137 & 21.70 & 605069 & 23520 & 3.88 & 69355 & 11.4 & 70879 & 6252 & 8.8 & 11568 & 16.32 & 1418745 & 44666 & & 281172 & 19.81 \\
\hline 2007-08 & 657414 & 8300 & 1.26 & 141134 & 21.46 & 611102 & 23295 & 3.81 & 74890 & 12.25 & 73145 & 5677 & 7.76 & 9480 & 12.96 & 1627451 & 54752 & 3.36 & 355200 & 21.82 \\
\hline 2008-09 & 35013 & 10428 & 1.52 & 148260 & 21.64 & 689946 & 20959 & 3.03 & 78570 & 11.38 & 65985 & 5770 & 8.74 & 11305 & 17.13 & 1743093 & 63920 & 3.66 & 348188 & 19.97 \\
\hline
\end{tabular}

Source: Agricultural Statistics of Pakistan (various issues).

Bar Diagram - 3

Bar Diagram Showing Major Crops Production Share of Pakistan and India in their Respective World Crop Production, 1995-96 to 2008-09

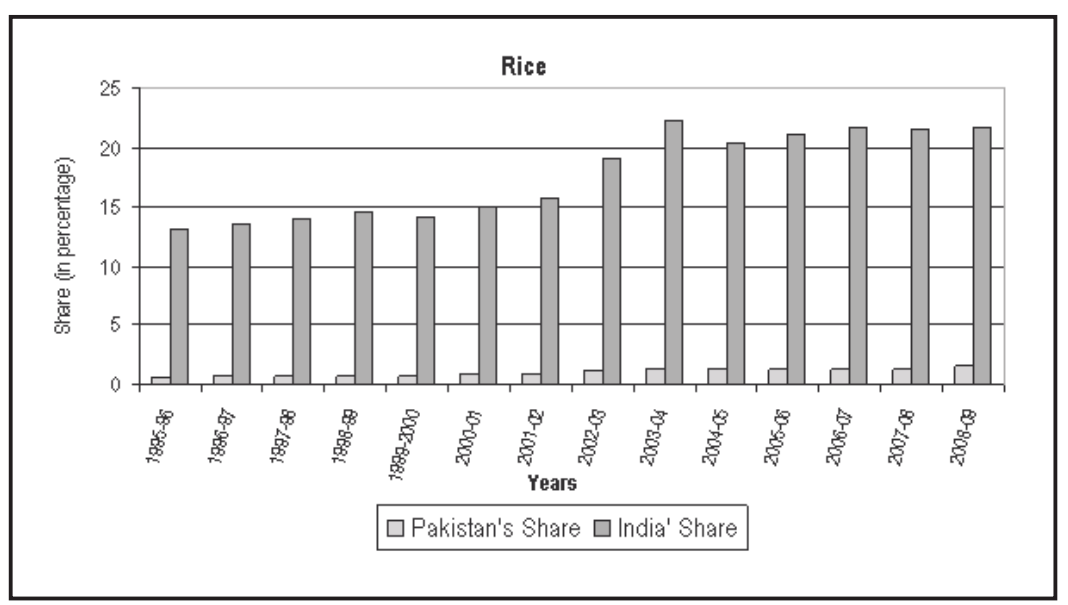



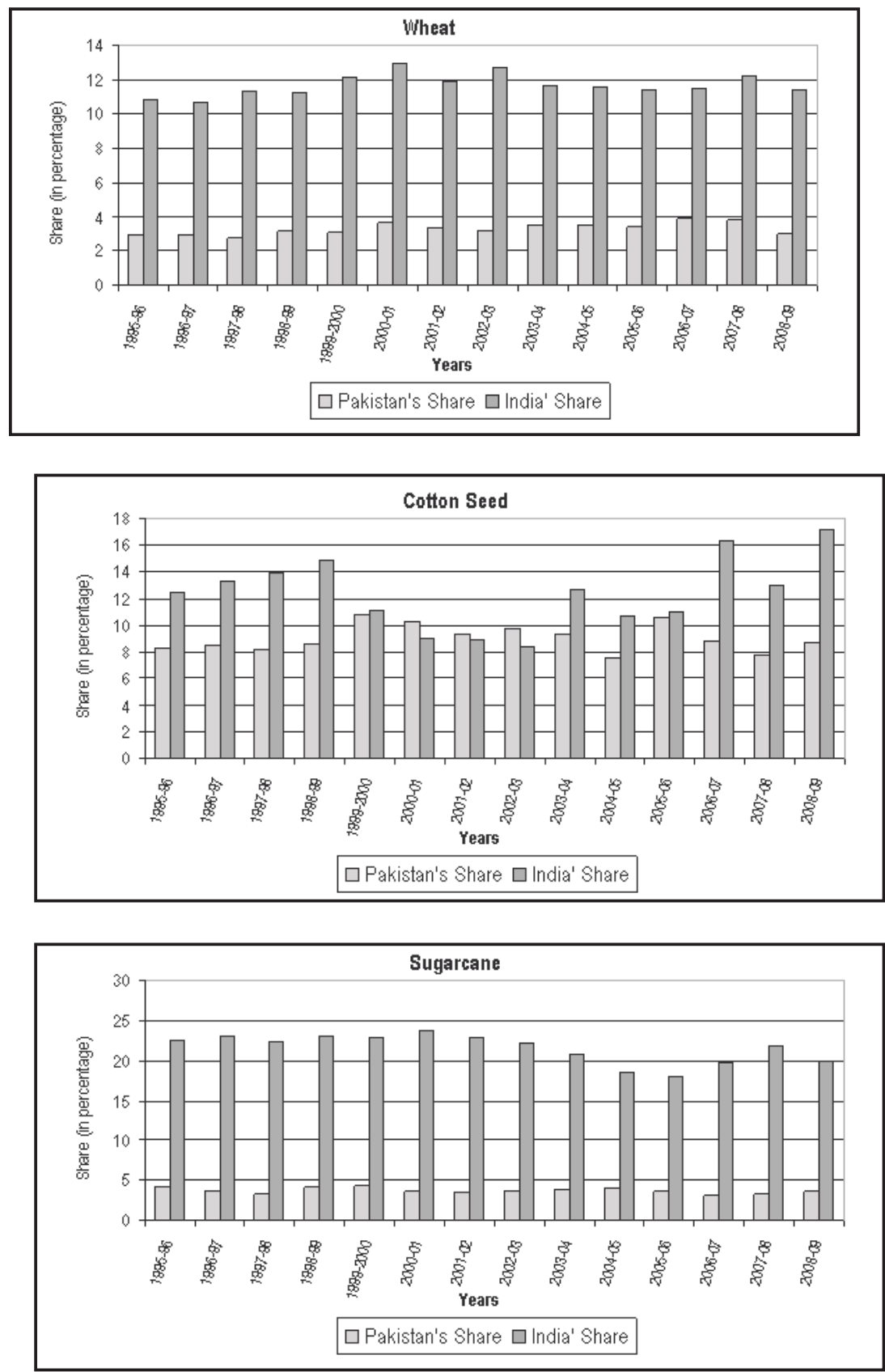

of implements, better control of flooded and draught land, and implementation of special programmes (especially in India) have played a vital role. India has low productivity per hectare of rice, wheat and cotton seed, as compared to world productivity per hectares (Table 4 and Figure 4). In most of the years India's productivity per hectares of wheat and sugarcane increased as compared to world productivity. This mainly 
occurred due to the utilization of HYVs, timely rainfall and better utilization of new farm technologies. India is the second largest producer of sugarcane in the world. In Pakistan the productivity per hectares of cotton seed in

\begin{tabular}{|c|c|c|c|c|c|c|c|c|c|c|c|c|}
\hline \multirow[b]{2}{*}{ Years } & \multicolumn{2}{|c|}{ Rice } & \multicolumn{4}{|c|}{ Wheat } & \multicolumn{3}{|c|}{ Cotton Seed } & \multicolumn{3}{|c|}{ Sugarcane } \\
\hline & \begin{tabular}{|c|} 
World \\
Productivit \\
y \\
(kg./hectar \\
es)
\end{tabular} & 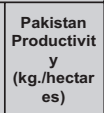 & \begin{tabular}{|c|} 
India \\
Productivit \\
(kg./hectar \\
(ks) \\
es
\end{tabular} & \begin{tabular}{|c|} 
World \\
Productivit \\
(kg./hectar \\
(es)
\end{tabular} & \begin{tabular}{|c|} 
Pakistan \\
Productivit \\
y \\
(kg./hectar \\
es)
\end{tabular} & \begin{tabular}{|c|} 
India \\
Productivit \\
(kg./hectar \\
(es)
\end{tabular} & $\begin{array}{l}\text { World } \\
\text { Productivit } \\
\text { (kg./hectar } \\
\text { es) }\end{array}$ & \begin{tabular}{|c|}
$\begin{array}{c}\text { Pakistan } \\
\text { Productivit } \\
\text { (kg./hectar } \\
\text { es) }\end{array}$ \\
\end{tabular} & \begin{tabular}{|c|} 
India \\
Productivit \\
(kg./hectar \\
es)
\end{tabular} & \begin{tabular}{|c|} 
World \\
Productivit \\
(kg./lhectar \\
es)
\end{tabular} & \begin{tabular}{|c|}
$\begin{array}{c}\text { Pakistan } \\
\text { Productivit } \\
\text { (kg./hectar } \\
\text { (es) }\end{array}$ \\
\end{tabular} & \begin{tabular}{|c|} 
India \\
Productivit \\
(kg./hectar \\
es)
\end{tabular} \\
\hline $1995-96$ & 3689 & 3689 & 1622 & 2536 & 2081 & 2480 & 1683 & 1596 & 907 & 62672 & 46747 & 76923 \\
\hline $1996-97$ & 3786 & 3786 & 1835 & 2523 & 2018 & 2484 & 1582 & 1584 & 805 & 62741 & 46968 & 68169 \\
\hline $1997-98$ & 3823 & 3823 & 1912 & 2676 & 2053 & 2679 & 1688 & 1584 & 867 & 64099 & 43521 & 66487 \\
\hline 1998-99 & 3747 & 3747 & 1870 & 2624 & 2238 & 2483 & 1561 & 1586 & 871 & 64423 & 50575 & 66919 \\
\hline $\begin{array}{l}1999- \\
2000\end{array}$ & 3894 & 3894 & 1928 & 2762 & 2170 & 2593 & 1623 & 1962 & 675 & 65479 & 47780 & 71270 \\
\hline $2000-01$ & 3885 & 3885 & 2050 & 2737 & 2491 & 2759 & 1676 & 1871 & 890 & 65451 & 45883 & 70769 \\
\hline $2001-02$ & 3870 & 3870 & 2021 & 2714 & 2341 & 2740 & 1710 & 1960 & 596 & 64927 & 45385 & 70617 \\
\hline $2002-03$ & 3581 & 3581 & 3019 & 2673 & 2262 & 2762 & 1730 & 1735 & 578 & 65313 & 48056 & 67370 \\
\hline $2003-04$ & 3970 & 3970 & 2955 & 2869 & 2388 & 2617 & 1929 & 1676 & 903 & 65532 & 47323 & 61116 \\
\hline $2004-05$ & 4019 & 4019 & 2991 & 2906 & 2586 & 2640 & 1949 & 1715 & 828 & 65597 & 49692 & 59707 \\
\hline $2005-06$ & 4084 & 4084 & 3174 & 2845 & 2519 & 2717 & 1992 & 2280 & 850 & 65781 & 48907 & 61952 \\
\hline 2006-07 & 4115 & 4115 & 3164 & 2857 & 2769 & 2619 & 2049 & 2033 & 1265 & 67973 & 49229 & 66928 \\
\hline 2007-08 & 4214 & 4214 & 3192 & 2985 & 2716 & 2671 & 2176 & 1859 & 1019 & 70696 & 53209 & 72555 \\
\hline 2008-09 & 4309 & 4309 & 3520 & 3086 & 2451 & 2802 & 2099 & 2046 & 1206 & 71510 & 51494 & 68877 \\
\hline
\end{tabular}

Source: Agricultural Statistics of Pakistan (various issues).

Figure - 4 Productivity per Hectare of Common Major Crops of Pakistan and India with Respective Crops World Productivity per Hectare, 1995-96 to 2008-09

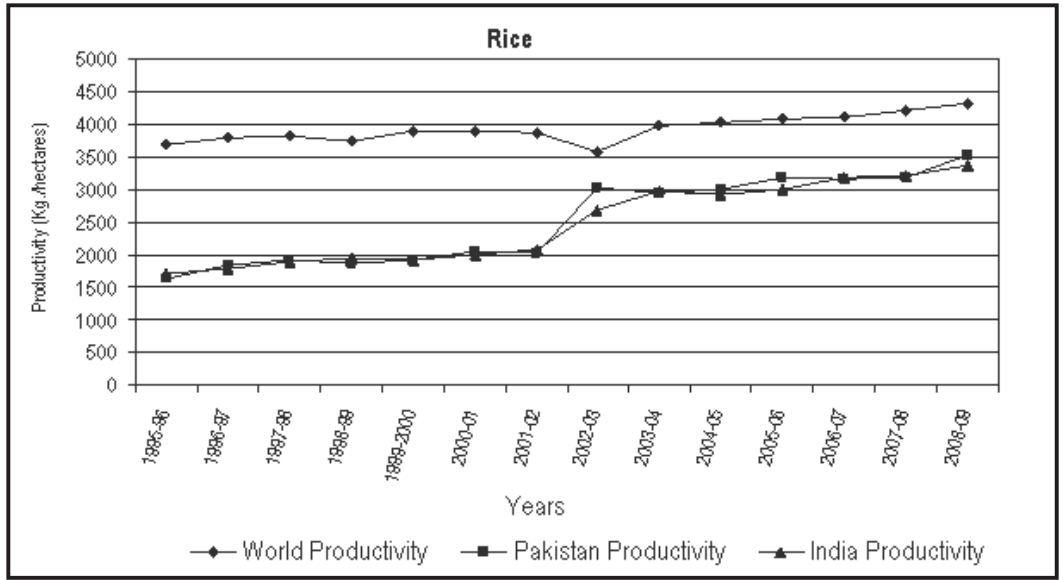



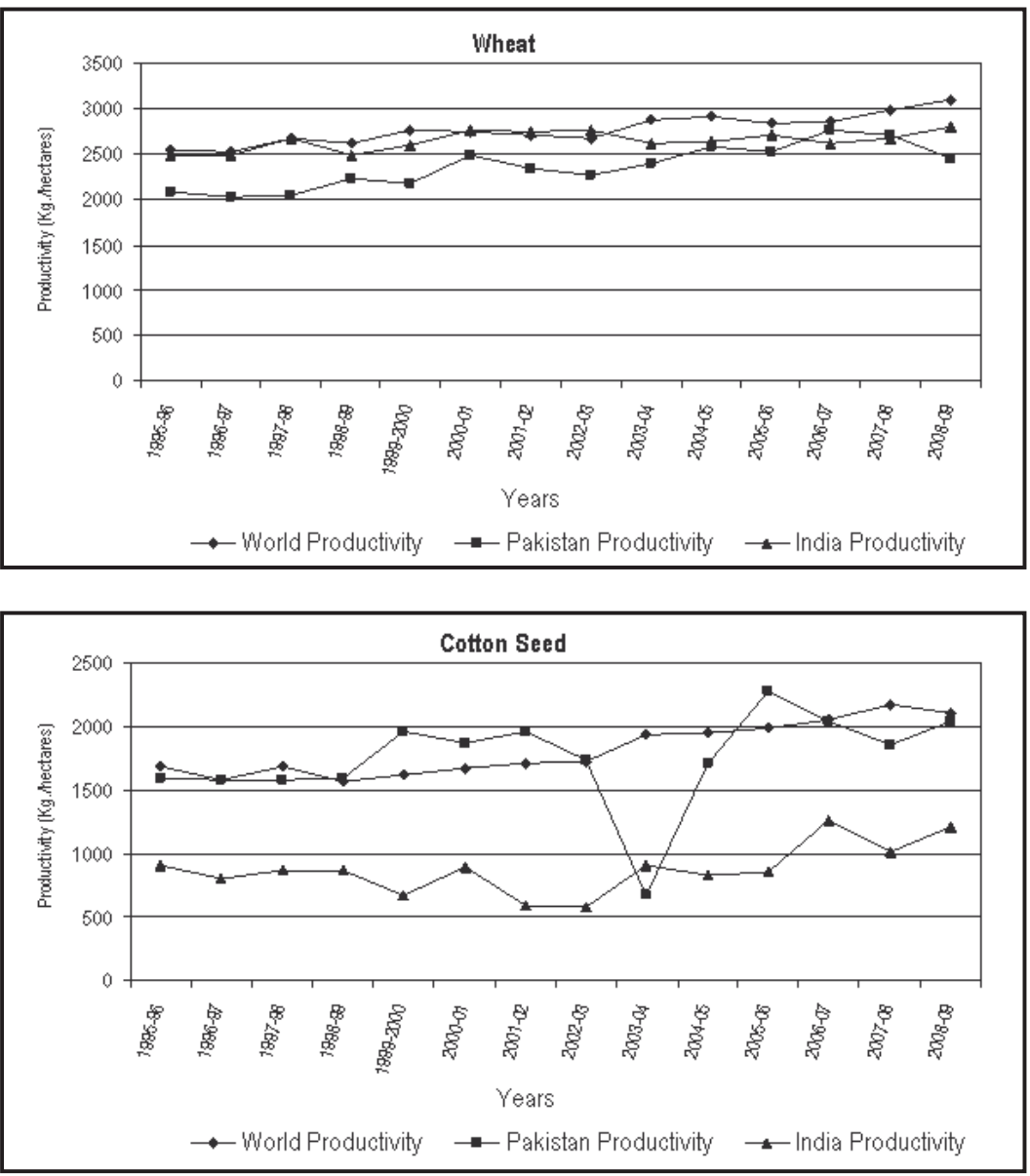

Figure - Productivity per Hectare of Common Major Crops of Pakistan and India with Respective Crops World Productivity per Hectare, 1995-96 to 2008-09

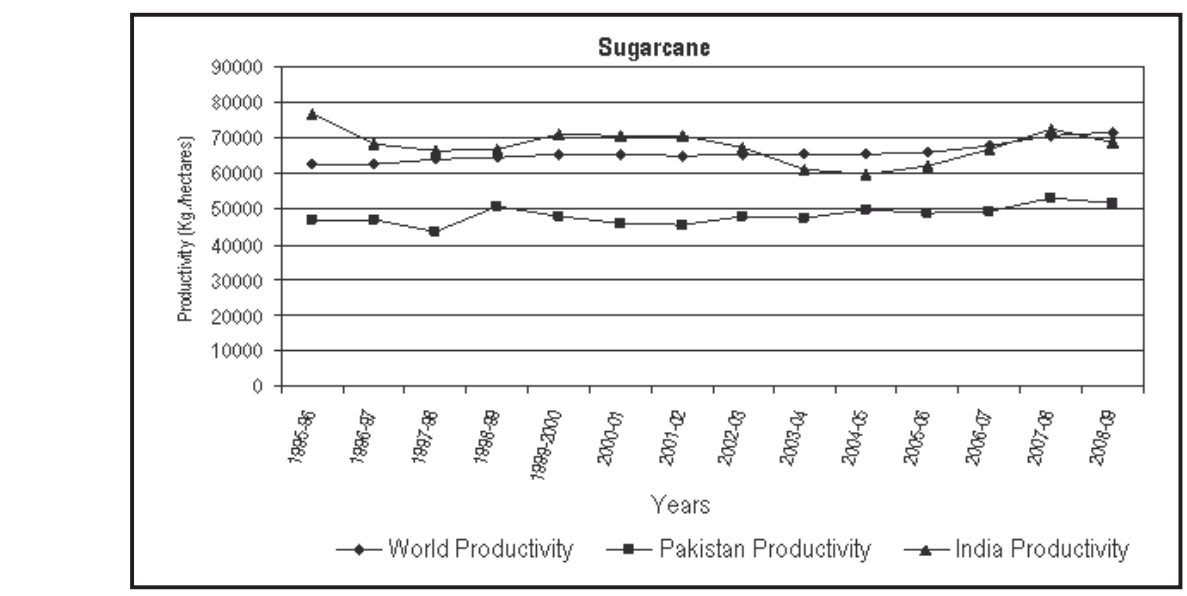


some of the years increased as compared to world cottonseed productivity. India's productivity per hectares of wheat and sugarcane is more as compared to Pakistan's productivity per hectares of these crops. Pakistan has more productivity per hectares of cottonseed as compared to India. Some of the yeas the productivity per hectare of rice was more as compared to India's productivity per hectare of this crop.

\section{Compound Growth Rates Of Area, Production And Productivity Of Major Crops Of Pakistan And India}

The annual compound growth rates of area, production and productivity have been estimated by using equation (1).

\section{Pakistan}

Table 5 shows that the production growth of rice significantly increased at the rate of 4.93 per cent per annum, wheat at 2.82 per cent per annum, cotton seed at 1.95 per cent per annum and sugarcane at 2.55 per cent per annum. The increase in the production growth of rice, wheat and sugarcane were due to increase in its area and productivity both but increase in its productivity contributed more as compared to increase in its area. While for cottonseed area and productivity both contributed equally. This clearly shows that new farm technology, favorable price incentive, better irrigation facilities, HYVs, and suitable climatic conditions have played a vital role in increasing the productivity growth.

Table - 5 Compound Growth Rates of Area, Production and Productivity of Major Crops in Pakistan and India, 1984-85 to 2008-09

\begin{tabular}{|c|c|c|c|c|c|c|}
\hline \multirow{2}{*}{ Crops } & \multicolumn{3}{|c|}{ Pakistan } & \multicolumn{3}{c|}{ Pakistan } \\
\cline { 2 - 7 } & Area & Production & Productivity & Area & Production & Productivity \\
\hline Rice & 1.44 & 4.93 & 3.49 & 0.33 & 3.90 & 3.57 \\
& $(11.60)^{\star}$ & $(11.59)^{\star}$ & $(9.16)^{*}$ & $(4.40)^{*}$ & $(15.11)^{*}$ & $(12.43)^{*}$ \\
\hline Wheat & 0.60 & 2.82 & 2.22 & 0.72 & 2.39 & 1.66 \\
& $(8.51)^{*}$ & $(16.59)^{*}$ & $(14.87)^{*}$ & $(7.71)^{*}$ & $(13.32)^{*}$ & $(10.97)^{*}$ \\
\hline Cottonseed & 0.99 & 1.95 & 0.97 & 0.96 & 0.98 & 0.16 \\
\hline Sugarcane & $(6.77)^{*}$ & $(5.56)^{*}$ & $(3.09)^{*}$ & $(5.95)^{*}$ & $(1.47)$ & $(0.27)$ \\
\hline & 1.17 & 2.55 & 1.38 & 1.72 & 0.50 & 0.68 \\
\end{tabular}

Note: * Significant at $1 \%$ level. Figures in parentheses are t values.

India

As shown in Table 5, the production growth rate of rice significantly increased at the rate of 3.90 per cent per annum and wheat at 2.39 per cent per annum. The increase in the production growth of rice and wheat was due to increase in its area and productivity but productivity contributed more than area. The increase in the productivity growth rate of sugarcane was mainly due to increase in its area growth rate rather than production growth rate. The significant increase in cottonseed area was insignificantly contributed by production and productivity growth rate. The increase in the productivity growth rate 
of rice and wheat was mainly due to good monsoon rainfall, better irrigation facilities, HYVs, and utilization of new farm technologies. Excessive rains and unexpected cyclones storms damaged the crop of cotton seed. There is a need to increase the production and productivity growth rate of cottonseed and sugarcane. This can be done through better price policy, agricultural credit, HYVs and better technology.

\section{Comparison Of Growth Rates Of Area, Production And Productivity Of Crops For Pakistan And India}

Table 5 clearly indicates that area growth rate of rice and cottonseed of Pakistan increased by more percentage per annum as compared to India. Similarly the area growth rate of wheat and sugarcane increased by more percentage for India as compared to Pakistan. As far as production and productivity growth rate are concerned it increased by more percentage for all the crops of Pakistan as compared to Indian crops.

Growth in productivity per hectares of any crop tells us that how far the farmers are utilizing new farm technologies in order to increase the production of the crop(s). It means that technology-based growth in productivity, favorable price incentives, better irrigation facilities, better and timely use of fertilizer and tractor, HYVs, utilization of new farm technologies, and good research and extension services played a vital role in increasing the productivity per hectare of all the crops of Pakistan. The production and productivity per hectare growth rate of crops clearly shows an edge to Pakistan over India.

\section{Association Between Growth In Major Crops Area And Productivity In Pakistan And India}

For clear understanding of the growth scenario, we discuss the association between growth in crops area and productivity (Table 6). All crops under study were classified into four types of association on the basis of growth rates of area and productivity. AApositive growth rate of area associated with positive growth rate of productivity. This would indicate that one crop is either replacing other crop or is grown in the newly cultivated area and the overall productivity of crop(s) increased. AB-positive growth rate of area associated with negative growth rate of productivity. BA-negative growth rate of area associated with positive growth rate of productivity. This would indicate that one major crop area has been replaced by other major crop or 
Table - 6 Association between Growth in Major Crops Area and Productivity in Pakistan and India 1984-85 to 2008-09

\begin{tabular}{|c|c|c|}
\hline Types of Association & Pakistan & India \\
\hline $\begin{array}{l}\text { AA: Positive area positive } \\
\text { productivity }\end{array}$ & $\begin{array}{l}\text { Rice, Wheat, Cottonseed, } \\
\text { Sugarcane }\end{array}$ & $\begin{array}{l}\text { Rice, Wheat, Sugarcane, } \\
\text { cotton seed }\end{array}$ \\
\hline $\begin{array}{l}\text { AB: Positive area negative } \\
\text { productivity }\end{array}$ & NIL & NIL \\
\hline $\begin{array}{l}\text { BA: } \\
\text { Negative area positive } \\
\text { productivity }\end{array}$ & NIL & NIL \\
\hline $\begin{array}{l}\text { BB: Negative area negative } \\
\text { productivity }\end{array}$ & NIL & NIL \\
\hline
\end{tabular}

Source: Taken from Table 5.

has gone out of cultivation and the productivity on the remaining area has increased. BB-negative growth rate of area associated with negative growth rate of productivity. It can seen from the Table that in AA category all the crops (rice, wheat, cottonseed and sugarcane) are falling for Pakistan and India.

We have also presented maximum and minimum productivity/yield rates per hectare of major crops of Pakistan and India in Table 7. The table concludes that the percentage difference in productivity rate of rice (56.59 per cent) is maximum and sugarcane (33.18 per cent) is minimum for Pakistan while in India it is rice ( 59.55 per cent) and sugarcane (23.91 per cent).

\section{Conclusion and Policy Implications}

The study analyzed and compared the growth rates of area, production and productivity of common major crops of Pakistan and India from 198485 to 200809 . The study reveals that in Pakistan the increase in the production growth rate of rice, wheat and sugarcane were due to increase in its area and productivity, but increase in its productivity contributed more as compared to increase in its area, while for cotton seed, the area and productivity both contributed equally. This was due to favorable price incentives, better utilization of technologies, better irrigation facilities, rising level of chemical fertilization application and HYVs etc. The study also confirms that in India the increase in the production growth rate of rice and wheat was due to increase in its area and productivity but productivity contributed more than area. The increase in the productivity growth rate of sugarcane was mainly due to increase in its area growth rate rather than production growth rate. The significant increase in cotton seed area was insignificantly contributed by production and productivity growth rate. The increase in the productivity growth rate of rice, wheat and sugarcane for India was mainly due to better monsoon rainfall, easy process of getting agricultural credit, HYVs and better utilization of new farm technologies. The study also confirms that area growth rate of rice and cottonseed of Pakistan increased by more percentage per annum as compared to India. The study also concludes that the area growth rate of wheat and sugarcane increased by more 
percentage for India as compared to Pakistan. As far as production and productivity growth rate are concerned, it increased by more percentage for all the crops of Pakistan as compared to Indian crops.

\section{Policy Implications}

- The study reveals that in Pakistan there is a need to increase the productivity growth rate of rice, wheat, cotton seed and sugarcane more because it is very difficult to increase the area of the crop due to heavy loss from water logging and salinity. The productivity growth rate can be increased by utilizing new farm technologies widely adapted to the agroclimatic conditions, favorable price incentives, extended irrigation system, timely application of fertilizer and pesticides, HYVs and better management services.

- $\quad$ The study also shows that in Pakistan the area growth rate of rice, wheat, cotton seed and sugarcane is not increasing. The government needs to apply current scientific methods in order to control water logging and salinity. Irrigation facilities also need to be increased.

- The study reveals that Indian farmers should increase the growth rate of area, production and productivity of cottonseeds and sugarcane. The decrease in these crops production growth was mainly due to excessive rains in Punjab and unexpected cyclonic storms in Andhra Pradesh. The increase in these crops production growth can be through favorable price incentives and timely as well as required monsoon rainfall.

- The study also reveals that in India, the crops production mainly depends upon monsoon rainfall. Climate is uncertain therefore the Indian government needs to improve their irrigation facilities. This can be done by making more dams and through installation of more tube-wells.

- $\quad$ The Indian farmers also need to increase the cropped area of most of their crops. Uncultivated land should be made cultivable with the help of new farm technologies (tractor and bulldozers). 


\section{References}

Ghaffar, C.M., Mustafa, G., and Qasim, A. (1996) 'Growth of Output and Productivity in Pakistan Agriculture: Trends, Sources and Policy Implications', The Pakistan Development Review, Vol.35, No.4, pp.527 536.

Government of Pakistan (various issues) Agricultural Statistics of Paksitan, Ministry of Food, Agriculture and Livestock, Islamabad.

Government of Pakistan, (2008 09) 'Economic Survey, Economic Advisers Wing, Finance Division, Islamabad.

Khan, M.H., and Mohsin, S.K. (1995) 'Agricultural Growth in China and sub-Saharan African Countries, The Pakistan Development Review, Vol.34, No.4, pp.429 456.

Khan, M.H., and Siddiqui, A.S. (1982) 'Growth and Fluctuations in the Output of Major Crops in Pakistan, 195051 to 1979 80', The Pakistan Development Review, Vol.21, No.2, ppp.149 158.

Mahendradev, S. (1987) 'Growth and Instability in Foodgrains Production: An Interstate Analysis', Economic and Political Weekly, Vol.22, N.39, pp.A 82 to A 92.

Mitra, A.K. (1990) 'Agricultural Production in Maharastra: Growth and Instability in the Context of New Technology', Economic and Political Weekly, Vol.25, No.52, pp.146 164.

Narain, D. (1977) 'Growth of Productivity in Indian Agriculture', Indian Journal of Agricultural Economics, Vol.32, No.1, pp.144.

Pal, S., and Siroshi, A.S. (1988) 'Sources of Growth and Instability in the Production of Commercial Crops in India', Indian Journal of Agricultural Economics, Vol.43, No.3, pp.

Parthasarathy, G. (1984) 'Growth Rates and Fluctuations of Agricultural Production: A District-Wise Analysis in Andhra Pradesh', Economic and Political Weekly, Vol.19, No.26, pp.A 74 to $A 84$.

Rao, V.M., and Deshpande, R.S. (1986) 'Agricultural Growth in India: A Review of Experiences and Prospects', Economic and Political Weekly, Vol.21, No.38 and 39, pp.A 101 to $A 112$.

Ray, S.K. (1983) 'An Empirical Investigation of the Nature and Causes for Growth and Instability in Indian Agriculture: 1950 80', Indian Journal of Agricultural Economics, Vol.38, No.4, pp.459 474.

Swant, S.D., and Achuthan, C.V. (1995) 'Agricultural Growth Across Crops and Regions: Emerging Trends and Patterns', Economic and Political Weekly, March 25, pp.A 2 to A 13.

Thakur, J., Singh, D.K., and Milon, R. (1988) 'An Analysis of Trends, Growth and Technological 
Development of Oilseeds in Bihar', Indian Journal of Agricultural Economics, Vol.43, No.3.

Wasim, M.P. (1999) 'Growth Rates and Fluctuations in Area, Production and Productivity: A Study of Major Crops in Sindh, Pakistan Economic and Social Review, Vol.37, No.2, Winter, pp.155 168. 
B I a n $\mathbb{k}$ 